\title{
Point Charge Dynamics Near a Grounded Conducting Plane
}

\author{
Kevin L. Haglin ${ }^{*}$ \\ Department of Physics and Astronomy, Saint Cloud State University, \\ 720 Fourth Avenue South, St. Cloud, MN 56301
}

(Dated: March 25, 2010)

\begin{abstract}
The classic image problem in electromagnetism involves a grounded infinite conducting plane and a point charge. The force of attraction between the point charge and the plane is identified using an equivalent-field picture of an image charge with opposite sign equidistant behind the plane resulting in a $1 / r^{2}$ force of attraction between the original charge and the plane. If the point charge is released from rest it will reach the plane in a time $\tau$. This time $\tau$ has not been calculated correctly up to now. Clarification of the inconsistency is presented along with a correct solution to the classic image problem. Other electromagnetism problems are mentioned with attractive $1 / r^{n}$ forces (where $n \geq$ $1)$. Such situations arise between a point charge and a line charge $(1 / r)$, between a line charge and a point dipole $\left(1 / r^{2}\right)$, between a point charge and a point dipole $\left(1 / r^{3}\right)$, and between a point dipole and a second point dipole $\left(1 / r^{4}\right)$.
\end{abstract}

\section{INTRODUCTION}

Point masses under the influence of Newtonian gravity and point charges under the influence of Coulombic attraction experience a $1 / r^{2}$ force. Particles in this picture will approach one another according to Newtonian (nonrelativistic) dynamics and if released from rest will reach the force center in a time that is typically shown to be proportional to the initial separation to the three-halves power. This type of solution appears in various places in textbooks as an application of gravitational forces 1, 2], and in electromagnetism textbooks surrounding the classic image problem [3 [5]. Unfortunately, the solution leading to this result is plagued with superluminal (i.e. faster than light speed) transport and hence cannot be formally correct.

In addition to relativistic effects, action-at-a-distance type forces require propagation of information about the force. News of changing particle positions must propagate to the other particle before any changes can be realized and the news travels at a fixed (finite) speed, namely, the speed of light. Care must be taken to properly account for these retardation effects. The ultimate effect is to extend the time it takes for the particle to reach the force center as compared with an assumption of instantaneous updating.

The electromagnetic category of problem is discussed in this paper, but the same formalism can be applied to the gravitational problem as well. The solution discussed below for problems of this type incorporates Einstein's special relativity and includes the effects of field retardation. In Section $\amalg$ the classic image problem is discussed and solved taking special relativity into account. After all, when particle separation approaches zero with an inverse squared force law, infinite kinetic energies are possible. This has all the earmarks of superluminal dynamics. Consistent results without any superluminal dynamics are presented in Section (III). The Liénard-Wiechart potentials are employed in Sect. (III) to take into account the retardation effects. Sect. (III) also includes a brief discussion to illustrate conditions under which the differences between the old (wrong) solution and the new (correct) solution are important. Discussion of the correspondence to analagous gravitational problems is included in Section (IV). A conclusion is included to summarize the main findings and to introduce other problems in electromagnetism where such effects could be important.

\section{THE CLASSIC IMAGE PROBLEM}

The classic image problem in electromagnetism involves a grounded infinite conducting plane and a point charge. The force of attraction between the point charge and the plane is identified using an equivalent picture of an image charge equidistant behind the plane of opposite sign. The field configuration in the half-space of the point charge is mathematically equivalent to the picture where an image charge of equal magnitude and opposite sign resides equidistant behind the plane. Hence, the field configuration of the point charge and the plane is mathematically equivalent to the the field between the point charge and the image charge in the space of interest.

If released from rest, the charge begins to move toward the plane, which also moves the image charge closer to the midpoint between the charge and the image. Consequently, the force of attraction between the charge and the image rapidly increases. The expectation in the textbooks is that Newton's 2nd law can be employed to write a second-order differential equation to be solved for $\overrightarrow{\mathbf{r}}(t)$. Typically, one is asked to show that the time to reach the plane is [4]

$$
\tau=\frac{\pi d}{q} \sqrt{2 \pi \epsilon_{0} m d}
$$

where $q$ is the charge, $m$ is the mass and $d$ is the initial

* klhaglin@stcloudstate.edu http://www.feynman.stcloudstate.edu/haglimbaration between the point charge and the plane. The 
permittivity of free space appears as $\epsilon_{0}$ which implies a particular system of units (SI).

Start with a point charge $q$ of mass $m$ released from rest a distance $d$ from an infinite grounded conducting plane (see Fig, 1). An image charge $q^{\prime}=-q$ placed a distance $d$ behind the plane will result in the correct boundary condition for the electric potential $V(0)=0$ and the correct field configuration for $x \geq 0$. Consequently, by uniqueness theorem arguments, one knows that the image configuration is mathematically equivalent to the original problem. As soon as the original charge moves, so too will the image charge (very much like two gravitating masses approaching one another; however, the image charge is not a physical charge so it does not introduce kinetic energy into the picture).

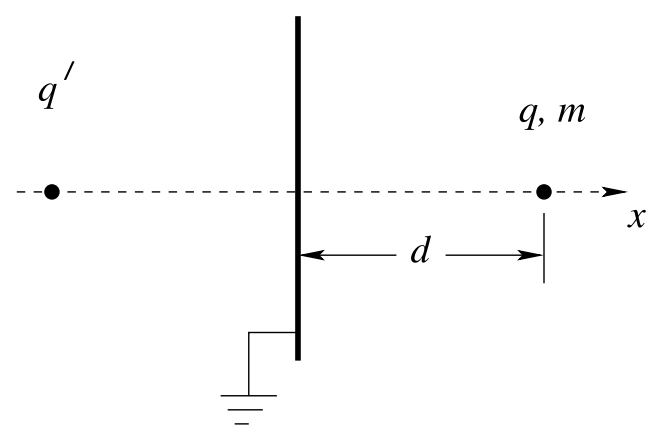

FIG. 1. Problem setup: Point charge released from rest a distance $d$ from a grounded infinite conducting plane.

The one-dimensional equation of motion under these conditions is (positive $x$ to the right)

$$
\frac{-q^{2}}{16 \pi \epsilon_{0} x^{2}}=m \frac{d^{2} x}{d t^{2}}
$$

where the initial conditions in time are $x(0)=d$ and $v(0)=0$, with the endpoint condition $x(\tau)=0$. Upon integrating once and applying the initial condition(s) one can write

$$
-\sqrt{\frac{q^{2}}{8 \pi \epsilon_{0} m}\left(\frac{1}{x}-\frac{1}{d}\right)}=v=\frac{d x}{d t} .
$$

Already here there is a problem! When the charge reaches the plane the value of $x$ becomes 0 . Therefore, the particle's speed becomes unbounded - clearly unphysical.

Still, if one proceeds to integrate once again to find an expression relating time $t$ and position $x$, one finds

$$
t=\tau\left[1-\frac{2}{\pi} \sin ^{-1}\left(\sqrt{\frac{x}{d}}\right)+\frac{2}{\pi} \sqrt{\frac{x}{d}} \sqrt{1-\frac{x}{d}}\right] .
$$

Setting $x=0$ identifies the time to reach the plane, but one must remember that particle's speed has become infinite at that moment.

How can this be corrected? The first step toward a complete solution is to use the relativistic expression for the particle's momentum $p=m \gamma v$. Then, changing the time derivative to a spatial derivative through $d t=v d x$, we can write

$$
\begin{aligned}
\frac{-q^{2}}{16 \pi \epsilon_{0} x^{2}} & =m \frac{d(\gamma v)}{d t}, \\
\frac{-q^{2}}{16 \pi \epsilon_{0} m} \frac{d x}{x^{2}} & =v \gamma^{3} d v .
\end{aligned}
$$

The Lorentz factor $\gamma=\left(1-v^{2} / c^{2}\right)^{-1 / 2}$ appears characteristically to modify the dynamics appropriately when the kinetic energy is not small compared with the rest energy. This expression can be integrated to find

$$
v=-c \frac{\sqrt{\ell^{2}\left(1-\frac{x}{d}\right)^{2}+2 \ell x\left(1-\frac{x}{d}\right)}}{\ell\left(1-\frac{x}{d}\right)+x},
$$

where a characteristic length $\ell=q^{2} /\left(16 \pi \epsilon_{0} m c^{2}\right)$ appears. Notice that $v(d)=0$ and $v(0)=-c$ (with the minus sign pointing to the direction) as it must to avoid superluminal transport. For a specific choice of charge, mass, and initial separation $[d /(c \tau)=0.5$, where $d /(c \tau)$ $=(1 / \pi) \sqrt{8 \ell / d}]$, a comparison is presented in Fig. 2 of the relativistic versus nonrelativistic expressions for the speed. For this particular configuration, the difference seems significant.

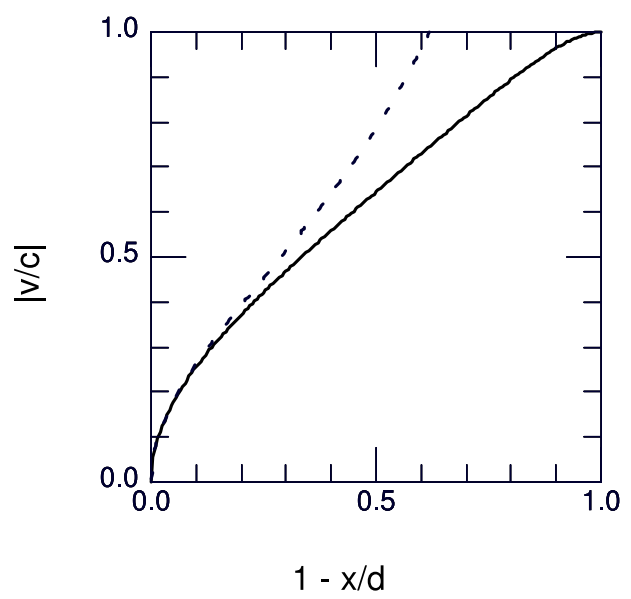

FIG. 2. Magnitude of velocity divided by $c$ versus distance traveled (that is, 1 minus the position divided by $d$ ) for a point charge released from rest near a grounded conducting plane. The solid curve (Eq. 6) describes relativistic dynamics and the dashed curve corresponds to nonrelativistic dynamics (Eq. 3).

The next task is clearly to integrate Eq. (6) to find an expression for $t$ as a function of $x$. Specifically, we must integrate

$$
c t=\int_{x}^{d} \frac{\ell\left(1-\frac{x^{\prime}}{d}\right)+x^{\prime}}{\sqrt{\ell^{2}\left(1-\frac{x^{\prime}}{d}\right)^{2}+2 \ell x^{\prime}\left(1-\frac{x^{\prime}}{d}\right)}} d x^{\prime} .
$$

The integral can be done in closed form and written as 


$$
\begin{aligned}
& \frac{t}{\tau}=\left(\frac{d}{c \tau}\right)^{2}\left(\frac{\pi}{4}\right)\left\{\left[\frac{1}{\sqrt{2}}\left(\sqrt{\frac{\ell}{d}}\right)\left(\frac{d}{\ell}-1\right)\right]\right. \\
& \cdot\left(1-\sqrt{\frac{1}{8}\left(\frac{\ell}{d}\right)\left(\frac{2 d}{\ell}-1\right)^{2}+1-\left(\frac{\sqrt{2} x}{d} \sqrt{\frac{d}{\ell}}-\frac{\sqrt{2}}{4}\left(\frac{\ell}{d}\right)\left(\frac{2 d}{\ell}-1\right)\right)^{2}}\right) \\
& +\left[\frac{\ell}{4 d}\left(\frac{2 d}{\ell}-1\right)\left(\frac{d}{\ell}-1\right)+1\right] \\
& \left.\left[\sin ^{-1}\left(\frac{\frac{x}{d} \sqrt{\frac{d}{\ell}}-\frac{1}{4} \sqrt{\frac{\ell}{d}}\left(\frac{2 d}{\ell}-1\right)}{\sqrt{\frac{1}{16}\left(\frac{\ell}{d}\right)\left(\frac{2 d}{\ell}-1\right)^{2}+\frac{1}{2}}}\right)-\sin ^{-1}\left(\frac{-\frac{1}{4}\left(\frac{2 d}{\ell}-1\right)}{\sqrt{\frac{1}{16}\left(\frac{2 d}{\ell}-1\right)^{2}+\frac{1}{2} \frac{d}{\ell}}}\right)\right]\right\} .
\end{aligned}
$$

A position-time analysis is now possible by comparing Eqs. (4) and (8). The comparison is shown in Fig. (3) with the solid curve representing relativistic dynamics and the dashed curve displaying the nonrelativistic expression. The same choice is made here for the dimensionless parameter as was made previously [namely, $d /(\mathrm{c} \tau)=0.5]$.

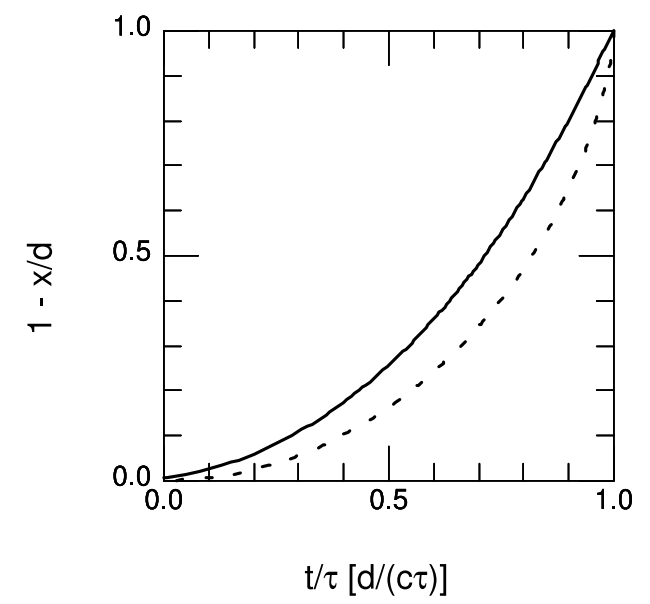

FIG. 3. Position (distance traveled in units of $d$ is " $1-x / d "$ ") versus time for a point charge released from rest near a grounded conducting plane. The dimensionless parameter is chosen to be $d /(c \tau)=0.5$. The solid curve corresponds to relativistic dynamics and dashed curve uses the nonrelativistic expression.

The slope of the tangent line is of course a measure of the magnitude of the instantaneous velocity. However, it cannot be read off directly since there is a factor of $d /(\tau c)$ in the ratio. And yet, notice that the dashed curve exhibits a slope for its tangent line near $x=0$ that is very large. Numerically the magnitude of the velocity exceeds 1 (in units of $c$ ) for $t / \tau \geq 0.62$ in the dashed curve. At $t / \tau=0.8$ the speed exceeds 2 , and it exceeds 4 at $t / \tau$ of 0.96 . Furthermore, we point out that the relativistically consistent curve displays a slope corresponding to a speed less than 1 throughout, only does the speed approach 1 at the point $x=0$.
Now that we have the expression for $t$ as a function of $x$, it is a straightforward exercise to take $x$ to zero and identify the time to reach the plane. By setting $x \rightarrow 0$, then $t \rightarrow T$, and we have,

$$
\begin{aligned}
\frac{T}{\tau}= & \left(\frac{d}{\tau c}\right)\left\{\frac{1}{2}-\left(\frac{d}{\tau c}\right)^{2} \frac{\pi^{2}}{16}\right. \\
& +\left[\frac{1}{\pi}\left(\frac{\tau c}{d}\right)+\left(\frac{d}{\tau c}\right) \frac{\pi}{16}+\frac{\pi^{3}}{128}\left(\frac{d}{\tau c}\right)^{3}\right] \\
& \left.\cdot\left[\frac{\pi}{2}-\sin ^{-1}\left(\frac{\frac{\pi^{2}}{8}\left(\frac{d}{\tau c}\right)^{2}-2}{\frac{\pi^{2}}{8}\left(\frac{d}{\tau c}\right)^{2}+2}\right)\right]\right\}
\end{aligned}
$$

where $\tau$ is the original nonrelativistic expression given by Eq. (11). We remark that if $c \rightarrow \infty$, the right hand side approaches 1 , so $T \rightarrow \tau$.

A comparison between the nonrelativistic expression for the time to reach the plane as compared with the fully relativistic expression is ultimately useful to be able to identify the conditions under which the original textbook expression fails. This comparison is deferred until after a discussion of the field-retardation effects.

\section{FIELD RETARDATION EFFECTS}

The discussion of the previous section improves upon the nonrelativistic analysis which is typically used to find the time to reach the plane. However, it too is lacking in some respects. When the charge is a distance $x$ from the plane and moving toward the plane, the force it experiences is different from a static Coulomb picture because the "electromagnetic news" travels at the speed of light. Hence, the electric field it experiences must be related to the electric and magnetic potentials at some earlier time (the so-called retarded time). Simply put, it takes a time $x / c$ for information on the particle dynamics to reach the plane. The charge configuration on the plane is updated (which means the image charge location is updated) and then that information requires a time $x / c$ to get back to the point charge. This type of physical circumstance 
is describable by the Liénard-Wiechart potentials for a moving point charge [6, 7].

Suppose the charge is located at position $x$ and moving with a velocity $-v$ (again, positive $x$ to the right). The general problem to calculate the retarded potentials is nontrivial, but when the motion is one-dimensional it simplifies significantly. In particular, the electric field experienced by the charge in this case is reduced by two powers of $\gamma$. It can therefore be written as

$$
E=\frac{-q}{4 \pi \epsilon_{0}}\left(\frac{1}{4 x^{2}}\right)\left(1-v^{2} / c^{2}\right) .
$$

The force drawing the charge toward the plane is therefore velocity dependent and it is reduced as compared with the static case. Consequently, the relativistic and properly time-delayed equation of motion is

$$
\frac{-q^{2}}{16 \pi \epsilon_{0} x^{2}}\left(1-\frac{v^{2}}{c^{2}}\right)=m \frac{d(\gamma v)}{d t} .
$$

Remarkably, this too can be integrated to find the velocity as a function of position. It becomes

$$
\frac{d x}{d t}=v=-c \sqrt{1-\left(\frac{x d}{x d+3 \ell(d-x)}\right)^{2 / 3}} .
$$

Another integral is required to identify a relationship between position and time in this relativistically consistent and properly casual description of the dynamics. Unfortunately, a closed form expression has not been found. Instead, the integral will be carried out numerically for the case of interest here. That is, the time $T$ to reach the plane will be computed and displayed graphically to help clarify the size of the effects. In particular, the time $T$ is

$$
\frac{T}{\tau}=\int_{0}^{1} \frac{\left(\frac{d}{\tau c}\right) d z}{\sqrt{1-\left(z /\left[z+\frac{3 \pi^{2}}{8}\left(\frac{d}{\tau c}\right)^{2}(1-z)\right]\right)^{2 / 3}}} .
$$

Time $T$ is normalized here by the original nonrelativistic time. A ratio of 1 indicates that the original nonrelativistic expression is completely satisfactory. Deviations from 1 indicate that relativistic effects and/or field retardation effects become important.

The time to reach the plane is again a function of the dimensionless parameter $d /(\tau c)$. In Fig. (4) the full solution is plotted as a function of $d /(\tau c)$. One must keep in mind that the dimensionless parameter is related to the charge, mass, and initial separation through

$$
\frac{d}{\tau c}=\frac{1}{\pi} \sqrt{\frac{8 \ell}{d}}=\frac{q}{\pi \sqrt{2 \pi \epsilon_{0} m c^{2} d}} .
$$

Graphical results indicate that so long as the dimensionless parameter $d /(\tau c)$ is less than roughly 0.1 , then the nonrelativistic expression for the time is completely adequate. For values of the parameter greater than 1 the relativistic and retardation effects become very important. What remains to be considered is to identify the physical situations that correspond to each of these regimes.

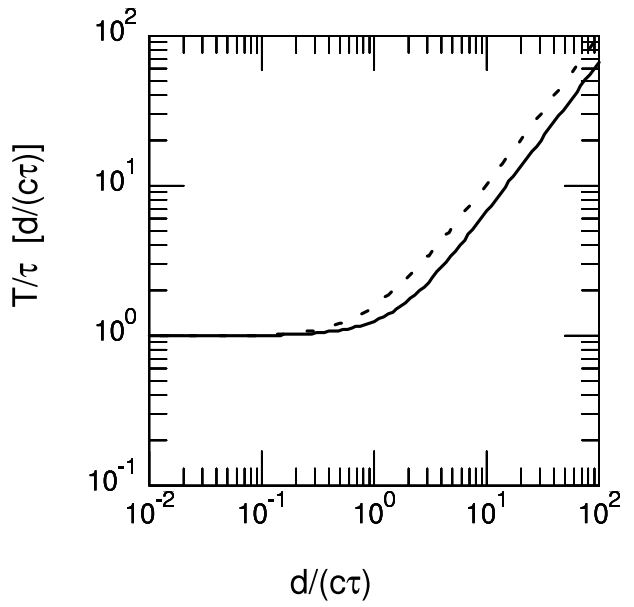

FIG. 4. Time to reach the plane versus scaled initial separation. Solid curve includes relativistic corrections, dashed curve includes relativistic correction plus retarded (LiénardWiechart) potentials.

\section{DISCUSSION}

There is of course some concern about using a solution for $\tau$ that involves superluminal transport. However, we have identified the crossover point where the relativistic expression must be used (see Fig 4 ). If a particular application falls to the left of the crossover point on Fig. (4), then the nonrelativistic formulation is completely adequate. It will be useful to consider a few example configurations to get a feel for this dimensionless parameter $d /(\tau c)$. Typical laboratory-type charges are microcoulombs, with typical masses in the milligram-togram range, and initial separations in the neighborhood of tens of centimeters.

For the typical configuration described above, the important parameter comes out to be of the order $10^{-6}$ as can be seen from the information in Table I. Consequently, the nonrelativistic solution is completely sufficient for this case. We emphasize that even though the nonrelativistic expression for the total time to reach the plane is valid, it does not mean the particle was completely nonrelativistic. Rather, the time spent moving relativistically was such a small part of the total time that relativistic corrections are unimportant. It is especially reassuring to notice that the number $10^{-6}$ is several orders of magnitude smaller than the region in Fig. (4) where the relativistic and retardation effects begin to be important.

Next we explore the case of an electron released from rest a distance $10 \mathrm{~cm}$ from a grounded conducting plane. Again from the table we extract the important dimensionless parameter for this case to be a number of the order of $10^{-8}$ (even smaller than the previous case!). This too is completely legitimately described by nonrelativistic formulae. The argument that saves the nonrelativis- 


\begin{tabular}{|l|l|l|l|}
\hline$q$ & $d$ & $m$ & $d /(\tau c)$ \\
\hline $1 \mu \mathrm{C}$ & $1(\mathrm{~cm})$ & 1 milligram & $\sim 10^{-6}$ \\
electron & $0.1($ meter $)$ & $m_{e}$ & $7.5 \times 10^{-8}$ \\
electron & $1(\mathrm{fm})$ & $m_{e}$ & $\sim 1$ \\
proton & $10(\mathrm{fm})$ & $m_{p}$ & $\sim 6 \times 10^{-3}$ \\
\hline
\end{tabular}

TABLE I. Comparison of different physical configurations and the resulting dimensionless parameter $d /(\tau c)$ which determines the region of applicability of relativity and fieldretardation effects.

tic expression is that even though the last tiny fraction of the trip to the grounded plane is superluminal, the charge spends so little time moving faster than light that the the deviation from the "correct" time is immeasurably small. So, once again, the nonrelativistic expression is sufficient.

Lastly, we investigate extreme cases where relativistic and retardation effects could be important. Consider an electron released from rest a distance 1 femtometer from a grounded conducting plane. The important parameter determining the dynamics is again the number $d /(\tau c)$ which comes out to be of the order 1 for this configuration. The nonrelativistic expression will fail noticeably for this case. Not only is relativity important for the dynamics, but field retardation effects are very important. A proton released $10 \mathrm{fm}$ from the plane results in a parameter $d /(\tau c)$ of $10^{-3}$.

Before concluding, it is useful to extend the solution presented above for the electrical charges to the case of two gravitating point masses. Consider two point masses $m$ initially separated by a distance $2 d$. We have immediately solved that problem as well using a correspondence between charge and mass, and between the inverse of the permittivity of free space and Newton's universal gravitational constant. The above dynamical expressions relating position, velocity and time hold for the case of gravity while the relevant dimensionless parameter becomes

$$
\frac{d}{\tau c}=\frac{\sqrt{2}}{\pi} \sqrt{\frac{m G}{d c^{2}}}
$$

where $G$ is Newton's universal gravitational constant. For most configurations this parameter is extremely small. Hence, once again, the nonrelativistic expression for the time to reach the center of mass is completely safe to use.

\section{CONCLUSION}

The classic image problem of a point charge near a grounded conducting plane invites the notion of a mathematical tool of replacing the grounded plane with a fictitious charge (a so-called image charge) which, together with the original charge, produces the same electric potential in the region of interest and respects the boundary conditions. The existence and uniqueness theorems indicate that the scalar potential and therefore the (vector) electric fields must be equivalent. It is a beautiful solution to an otherwise difficult problem.

The follow-up problem involves computing the time it takes the charge to reach the plane when the charge is released from rest. A straightforward (although incorrect) analysis from nonrelativistic classical mechanics results in an expression for the time which is proportional to the initial separation to the three-halves power, also roportional to the square root of the mass, and inversely proportional to the charge. We have pointed out in this article the serious formal flaw in that proposed solution. The flaw is linked to the observation that the velocity of the charged particle exceeds the speed of light for a small portion of its trip toward the grounded plane. It is very important to sort out when the solution is still valid and acceptable, and when it is noticeably inadequate. This has been the purpose of our article.

We have shown that the relativistic version of the problem is completely tractable and results in subluminal dynamics which changes the expression for the time to reach the plane by monotonically increasing the result as a function of the dimensionless parameter $d /(\tau c)$.

A more sophisticated approach to point charge dynamics near a grounded conducting plane must include fieldretardation effects. This has also been added to the solution discussed in this paper. Using time-delayed fields (Liénard-Wiechart potentials) increases further the time required for the particle to reach the plane. Both effects, relativistic and retardation influences, are easily understood in terms of their tendency to increase the time for the particle to reach the plane.

Finally, we briefly mention other problems where these same effects could be important. First, a point charge $q$ of mass $m$ near a fixed line charge $-\lambda$ experiences a force that is proportional to $1 / r$. The nonrelativistic time to reach the line charge can be computed as [8] $\tau=$ $\pi d \sqrt{\epsilon_{0} m /(\lambda q)}$. However, the charge goes superluminal as it reaches the line charge. A point dipole $p$ of mass $m$ near a fixed line charge $-\lambda$ experiences a $1 / r^{2}$ force. Using nonrelativistic dynamics again we find the time to reach the line charge is $\tau=(\pi d / 4) \sqrt{\pi \epsilon_{0} m d /(p \lambda)}$. Furthermore, a point charge $q$ of mass $m$ near a point dipole $p$ of mass $m$ will experience an inverse cube force $\left(1 / r^{3}\right)$. The nonrelativistic time for the two to meet at the midpoint (each of which is initially a distance $d$ away from the midpoint) is $\tau=d^{2} \sqrt{2 \pi \epsilon_{0} m /(p q)}$. And lastly, two identical point dipoles $p$ with mass $m$ will experience a force proportional to $1 / r^{4}$. The nonrelativistic expression for the time to reach the midpoint is $[9] \tau=(2 / 3) \kappa d^{2}$ $\sqrt{\pi \epsilon_{0} m d} / p$, where the constant $\kappa$ can be computed as

$$
\kappa=\int_{0}^{\pi / 2}\left[\sin ^{2 / 3} \theta\right] d \theta=1.12 \ldots
$$

In each case above, analytic or numerical expressions for velocity as a function of position and position as a function of time can be identified. Relativistic corrections and field retardation effects are equally readily obtainable. For increasingly steeper $r$-dependence, one might 
expect that the effects could be increasingly more important. The small-distance behavior determines the relative importance of the effects, and hence the dipole-dipole is the most sensitive to these effects.

\section{ACKNOWLEDGMENTS}

This work was supported in part by the National Science Foundation under grant No. PHY-0555521.
[1] R. Resnick, D. Halliday and K.S. Krane, Physics, Vol. 1, Fifth Edition, J. Wiley \& Sons, Inc., 328 (2002).

[2] S.T. Thornton and J.B. Marion, Classical Dynamics of Particles and Systems, Fifth Ediction, Brooks/Cole, 205 (2004).

[3] B.F. Bayman and M. Hammermesh, A Review of Undergraduate Physics, J. Wiley \& Sons, Inc., 117 (1986).

[4] D.J. Griffiths, Introduction to Electrodynamics, Third Edition, Prentice Hall Inc., 155 (1999).

[5] S. Palit, Principles of Electricity and Magnetism, Alpha Science International Ltd., 48 (2005).

[6] J.D. Jackson, Classical Electrodynamics, Third Edition, 661 (1999).

[7] D.J. Griffiths, Introduction to Electrodynamics, Third Edition, Prentice Hall Inc., 435 (1999).

[8] The expression to find $\tau$ is proportional to $I=$ $\int_{0}^{\infty} d \eta \sqrt{\eta} e^{-\eta}$. Square the integral, which becomes a dou- ble integral over $\eta$ and $\nu$. Change variables to $u=\eta+\nu$ and $v=\eta-\nu$. The area element $d \eta d \nu$ becomes $2 d u d v$. The resulting integral is soluble with the result coming out as $I=\sqrt{\pi} / 2$.

[9] In this case, $\tau$ is proportional to $\kappa=$ $\int_{0}^{\infty} d \eta e^{-(5 / 3) \eta} / \sqrt{1-e^{2 \eta}}$. Expanding and integrating term by term gives

$$
\begin{aligned}
\kappa & =3 \sum_{n=1}^{\infty} \frac{(2 n-3) ! !}{(6 n-1)(n-1) ! 2^{n-1}} \\
& =3\left\{\frac{1}{5}+\frac{1}{22}+\frac{3}{136}+\frac{15}{1104}+\ldots\right\} \\
& =1.12 \ldots
\end{aligned}
$$

The same result comes from numerically integrating Eq. (16). 